\title{
Radioterapia Mediastínica e Lesão Ostial de Tronco de Coronária Esquerda
}

\author{
Edgar Guimarães Victor, Giordano Bruno de O liveira Parente
}

Recife, PE

\begin{abstract}
Doença cardíaca isquêmica é uma complicação rara e, só recentemente reconhecida, de irradiação mediastínica para tratamento de tumores nesta região. É relatado caso de uma mulher de 51 anos com angina do peito, rapidamente progressiva, em que o achado angiográficofoi representado por lesão suboclusiva ostial de tronco de coronária esquerda. A história pregressa era marcada por uso de radioterapia para tratamento de linfoma Hodgkin mediastínico, com íntima relação com ventrículo direito, ressecado cirurgicamente e tratado em seqüência com irradiação e quimioterapia, dois anos antes. A indução de estenoses coronarianas nesses pacientes pode ser dependente ou não de aterosclerose focal e é mediada, principalmente, por espessamento intimal decorrente de fibrose tissular, sem que haja alteração na camada média e com predileção pelas porções proximais (ostiais) das artérias principais. $O$ reconhecimento desta condição (radioterapia torácica), como fator isolado e independente para doença coronariana, deve ser considerada na programação de medidas para prevenção, detecção e tratamento precoce.
\end{abstract}

A doença arterial coronariana constitui condição de alta prevalência e de reconhecida repercussão clínica, sendo, na sua maioria, decorrente de lesão arterial aterosclerótica. Determinadas situações, consideradas inusitadas, como a presença de lesões focais e ostiais de tronco coronariano, particularmente quando em pacientes com pouco ou nenhum fator de risco cardiovascular, devem alertar o clínico para possibilidades não usuais implicadas na gênese do processo. Podemos citar como exemplo, a radioterapia mediastínica, procedimento que, induzindo potencialmente alterações na estrutura microscópica do endotélio vascular coronariano $^{1}$, pode ocasionar lesões obstrutivas das artérias coronárias ${ }^{2}$ e isquemia miocárdica, processo que pode ocorrer anos após o uso da radiação ionizante.

Hospital das Clínicas - Universidade Federal de Pernambuco

Correspondência: Edgar Guimarães Victor - Av. Beira Mar, 138/101

Cep 54400-010 - Jaboatão dos Guararapes, PE - E-mail: evictor@truenet.com.br Recebido em 29/11/2002

Aceito em 11/8/03

\section{Relato do Caso}

Mulher de 51 anos, dentista, há 30 dias com queixa de dor retroesternal com irradiação para fúrcula e região interescapulovertebral direita, inicialmente, a esforços habituais, progressiva nos últimos 5 dias da avaliação, quando, após esforço físico moderado teve dor de grande intensidade com irradiação para membro superior direito e sensação de morte iminente. Negava sintomas concomitantes, ou qualquer outra sintomatologia cardiovascular. Dislipidemia, tratada com atorvastatina, e menopausa, em uso de reposição hormonal, constituíam fatores isolados de risco há 6 anos. Não havia evidência de hipertensão, diabetes ou tabagismo ou antecedentes familiares.

A cerca de dois anos houve o diagnóstico de linfoma Hodgkin mediastínico com compressão ventricular direita, ressecado cirurgicamente, tendo feito, na ocasião, quimioterapia (adriamicina + vinblastina) por 6 meses, além de 40 sessões de radioterapia.

O exame físico evidenciava pressão arterial de 125x80mmHg, 72bpm, pulsos simétricos, pulmões normais, ritmo regular com $4^{\mathrm{a}}$ bulha presente, sem sopros.

A radiografia de tórax, quando do diagnóstico de linfoma, demonstrava alargamento do mediastino (fig 1). $\mathrm{O}$ eletrocardiograma e ecocardiograma naquela ocasião, eram normais, à exceção de sinais ecocardiográficos de massa tumoral comprimindo a parede anterior do ventrículo direito. $\mathrm{O}$ eletrocardiograma atual (fig 2) evidenciou ritmo sinusal, 72ciclos/min, discreto infradesnivelamento ST com onda T negativa em DI, AVL, V3 a V6 e o ecocardiograma demonstrou leve hipocinesia ântero-septal.

Na vigência deste quadro clínico, a paciente foi inadvertidamente submetida a um teste ergométrico. No $2^{\circ}$ minuto de esforço do protocolo de Bruce ocorreram dor precordial intensa e taquicardia ventricular monomórfica não-sustentada, com infradesnivelamento do segmento ST de até 3,3mm em MC5, V5 e V6, mantidas até o $12^{\circ}$ minuto da recuperação (fig 3).

A coronariografia mostrou lesão ostial suboclusiva de tronco de coronária esquerda, demais artérias coronárias isentas de aterosclerose (fig. 4). No mesmo dia a paciente foi submetida à revascularização cirúrgica, utilizando-se enxer- 


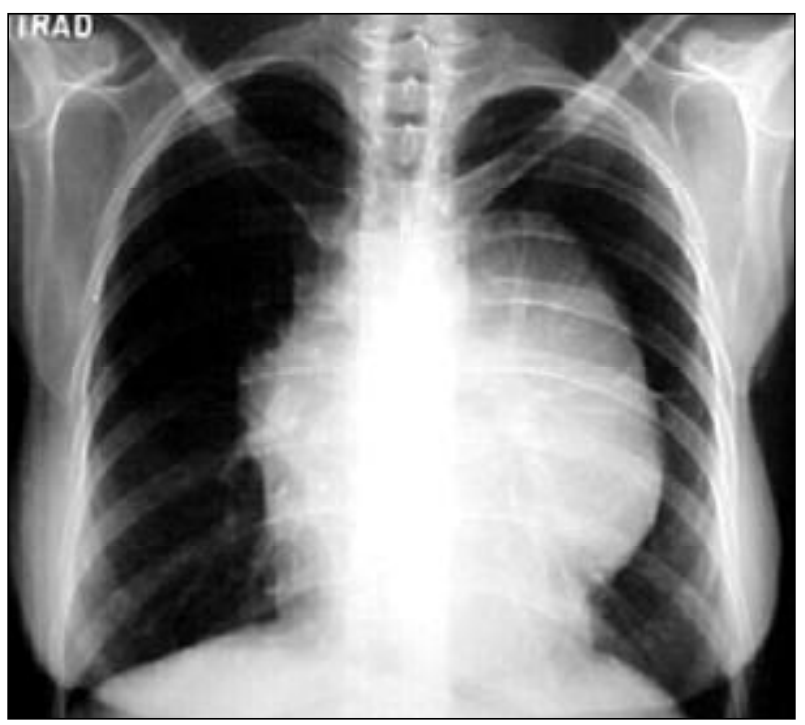

Fig.1 - Radiografia de tórax demonstrando alargamento mediastínico.

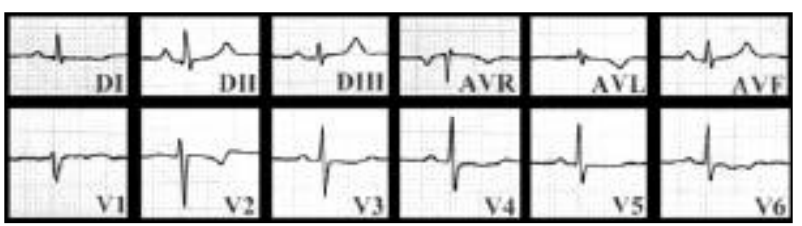

Fig. 2 - Eletrocardiograma de 12 derivações demonstrando inversão de onda T (em parede lateral).

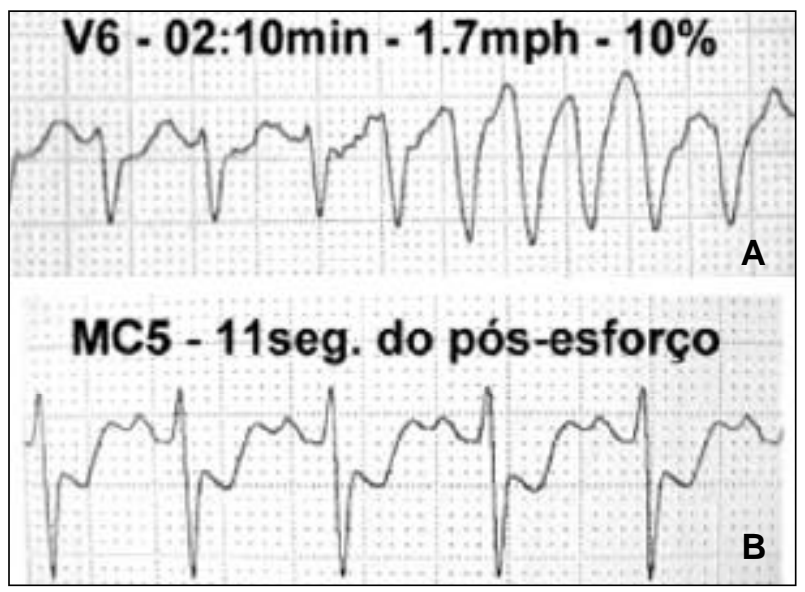

Fig.3 - Eletrocardiograma registrado durante o esforço demonstrando taquicardia ventricular (A) e infradesnivelamento do segmento ST (B).

tos venosos. Não houve intercorrências clínicas no transoperatório e a evolução atual não registra eventos mórbidos.

\section{Discussão}

A obstrução ostial em tronco de artéria coronária esquerdaé um achado raro, correspondendo 0,13 a $2,7 \%$ dos estudos angiográficos ${ }^{1}$, associada na maioria dos casos à doença co-existente em múltiplos vasos. Na literatura, o achado de lesão ostial em tronco da artéria coronária esquerda, na

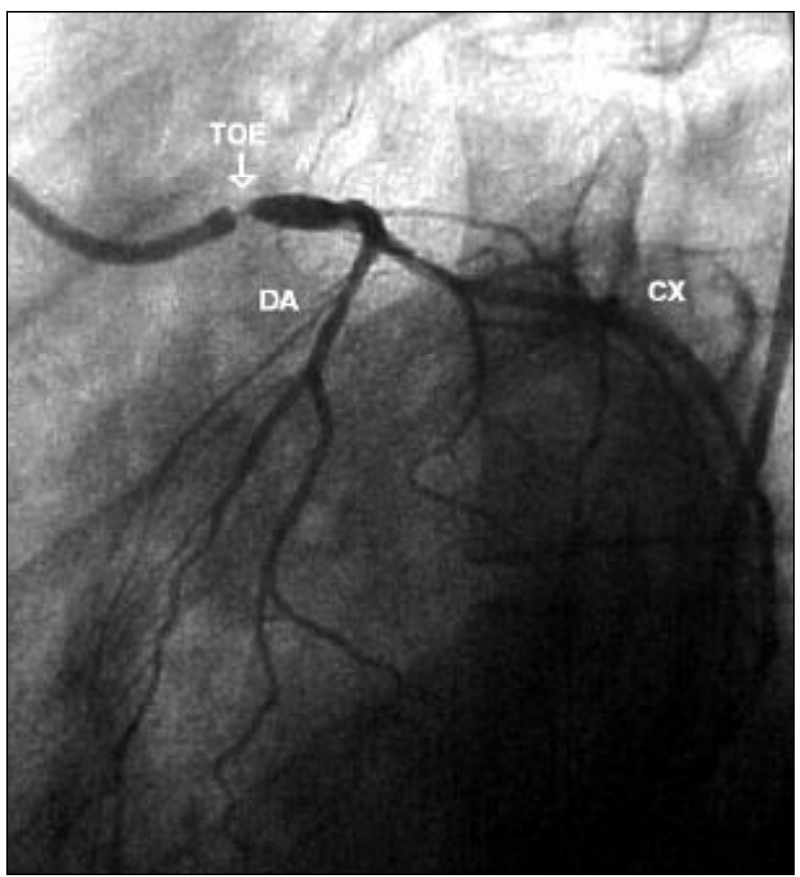

Fig.4 - Cineangiocoronariografia em (seta); (DA: desecendente anterior; CX: circunflexa); OAE demonstrando obstrução crítica ostial de tronco do coronária esquerda.

ausência de outras lesões, pode ser atribuída a diversas etiologias, como congênita, secundária a arterites (Takayasu, luética), displasia fibromuscular, iatrogênica (após troca valvar aórtica), idiopática e secundária à fibrose intimal focal por radioterapia dirigida ao mediastínico.

As principais complicações cardíacas de pacientes submetidos à radioterapia são a pericardite e miocardite, sendo mais raro o envolvimento valvular ${ }^{2}$, infundíbulo-pulmonar $^{3}$ e coronariano. Neste último caso, caracteristicamente, os pacientes que desenvolvem os sintomas em média 5 anos após à exposição, são relativamente jovens, com poucos ou nenhum fator de risco para doença aterosclerótica ${ }^{4}$, na grande maioria (85\%), com linfoma mediastínico do tipo Hodgkin, sendo os sintomas iniciais a angina, insuficiência cardíaca e infarto, e até mesmo, morte súbita. A presença de hipercolesterolemia, uso concomitante de agentes quimioterápicos (principalmente doxorrubicina) e grau de irradiação $0^{5}$ aumentam o risco para desenvolvimento de doença coronariana.

Em revisão de casos de doença coronariana secundária à radioterapia, estima-se uma incidência em torno de $18 \%{ }^{5}$, sendo $16 \%$ representados por lesões ostiais isoladas ${ }^{6}$. Nesses casos, o aspecto histopatológico característico é o espessamento localizado da camada íntima, sem evidência de dano na camada média ou depósito lipídico sugestivo de deposição ateromatosa ${ }^{1,7}$. Uma outra apresentação, também mencionada na literatura, seria a da própria doença aterosclerótica acelerada, associada ao espessamento focal intimal ${ }^{7}$. Nesses pacientes outra particularidade é a grande incidência de dano concomitante das artérias mamárias, tornando assim o seu uso durante a revascularização cirúrgica proibitivo e associado a maus resultados ${ }^{8}$.

Procedimentos terapêuticos e diagnósticos, utilizando 
radiação ionizante sobre o tórax, em especial no tratamento de tumores mediastínicos ou mesmo neoplasias de mama, devem ser reconhecidos como fator de risco independente para o aparecimento de doença coronariana. Nestas circunstâncias o acometimento arterial é frequentemente grave, lesando segmentos proximais.

Embora contemple outra forma de aplicação de radiação, o uso de braquiterapia, tratamento de escolha para reestenose intrastent, até o recente emprego dos stents revestidos com substâncias antiproliferativas ${ }^{9}$, pode ser rela- cionado ao desenvolvimento de trombose tardia intrastent, possivelmente em consequência da endotelização incompleta ou comprometedora da função endotelial. A reestenose das margens, istoé, das bordas dos segmentos irradiados, é responsável por até $40 \%$ da reestenose pósbraquiterapia ${ }^{10}$.

Nessas circunstâncias, o alerta para a ação danosa dessa forma de tratamento recomenda medidas de profilaxia e testes periódicos de triagem nos pacientes submetidos à radiação diagnóstica e/ou terapêutica sobre o tórax.

\section{Referências}

1. Grollier G, Commeau P, Mercier V, et al. Post-radiotherapeutic left main coronary ostial stenosis: clinical and histological study. Eur Heart J 1988; 9: 567-70.

2. Gonzaga AT, Antunes MJ. Post-radiation valvular and coronary artery disease. J Heart Valve Dis 1997: 6; 219-21.

3. Horimoto M, Igarashi K, Takenaka T, et al. Pulmonary infundibular stenosis, coronary artery disease, and aortic regurgitation caused by mediastinal radiation. Am Heart J 1993: 126; 1002-5.

4. Orzan F, Brusca A, Conte MR, et al. Severe coronary artery disease after radiation therapy of the chest and mediastinum: clinical presentation and treatment. $\mathrm{Br}$ Heart J 1993: 69; 496-500.

5. Annest LS, Anderson RP, Li W, et al. Coronary artery disease following mediastinal radiation therapy. J Thorac Cardiovasc Surg 1983: 85; 257-63.

6. Pilliere R, Luquel L, Brun D, et al. Ostial stenosis of the left main coronary artery after mediastinal radiotherapy. A propos of a case. Arch Mal Coeur Vaiss 1991: 84; 869-72.

7. Virmani R, Farb A, Carter AJ, et al. Pathology of radiation-induced coronary artery disease in human and pig. Cardiovasc Radiat Med 1999: 1; 98-101.

8. Khan MH, Ettinger SM. Post mediastinal radiation coronary artery disease and its effects on arterial conduits. Catheter Cardiovasc Interv 2001: 52; 242-8 .

9. Sousa E, Costa R, Abizaid A, et al. Lack of neointimal proferation of sirolimuscoated stents in human coronary arteries. A quantitative coronary angiography and three-dimensional intravascular ultrassound study. Circulation 2001: 103; 192-5.

10. Sabate M, Costa M, Kozuma K, et al. Geographic miss a cause of treatment failure in radio-oncology applied to intracoronary radiation therapy. Circulation 2000: $101 ; 2467-71$. 